\title{
Serum triglyceride levels in relation to high-density lipoprotein cholesterol (TG-HDL) ratios as an efficient tool to estimate the risk of sleep apnea syndrome in non-overweight Japanese men
}

\author{
Yuji Shimizu $^{1} \cdot$ Hiroyuki Yoshimine $^{2} \cdot$ Mako Nagayoshi $^{1} \cdot$ Koichiro Kadota $^{1}$. \\ Kensuke Takahashi $^{2} \cdot$ Kiyohiro Izumino $^{3}$ - Kenichiro Inoue ${ }^{3}$. Takahiro Maeda ${ }^{1,4}$
}

Received: 7 January 2016/Accepted: 6 April 2016/Published online: 19 April 2016

(c) The Japanese Society for Hygiene 2016

\begin{abstract}
Objectives Several studies have reported the association between sleep apnea syndrome and insulin resistance. Being overweight is known risk factor both for sleep apnea syndrome and insulin resistance. However, no studies have reported on the association between serum triglyceride levels in relation to high-density lipoprotein cholesterol (TG-HDL) ratios (a marker of insulin resistance) and sleep apnea syndrome accounting for body mass index (BMI) status.

Methods Subjects for the present cross-sectional study consisted of 1,528 men aged 30-69 years undergoing pulse oximetry at a sleep disorders clinic for sleep apnea syndrome. Sleep apnea syndrome was diagnosed as a $3 \%$ oxygen desaturation index (ODI) of $\geq 15$ events/h.

Results Among study participants, 241 men were diagnosed with sleep apnea syndrome. Independent of classical cardiovascular risk factors, TG-HDL was significantly positively associated with sleep apnea syndrome in participants with a BMI $<25 \mathrm{~kg} / \mathrm{m}^{2}$, but not in participants with a $\mathrm{BMI} \geq 25 \mathrm{~kg} / \mathrm{m}^{2}$. The multivariable adjusted odds ratio $(\mathrm{OR})$ and $95 \%$ confidence interval $(95 \% \mathrm{CI})$ of sleep
\end{abstract}

Yuji Shimizu

simizicyuu@yahoo.co.jp

1 Department of Community Medicine, Nagasaki University Graduate School of Biomedical Science, Nagasaki, Japan

2 Department of Respiratory Medicine, Inoue Hospital, Nagasaki, Japan

3 Shunkaikai, Inoue Hospital, Nagasaki, Japan

4 Department of Island and Community Medicine, Nagasaki University Graduate School of Biomedical Science, Nagasaki, Japan apnea syndrome per Log TG-HDL was 2.03 (95\% CI: 1.36-3.03) for a BMI $<25 \mathrm{~kg} / \mathrm{m}^{2}$ and 1.23 (95\% CI: $0.89-1.70$ ) for a BMI $\geq 25 \mathrm{~kg} / \mathrm{m}^{2}$.

Conclusions An independent positive association between TG-HDL levels and risk of sleep apnea syndrome was observed in participants with a BMI of $<25 \mathrm{~kg} / \mathrm{m}^{2}$, but not in participants with a BMI $\geq 25 \mathrm{~kg} / \mathrm{m}^{2}$. TG-HDL levels could be an efficient tool to estimate the risk of sleep apnea syndrome in non-overweight Japanese men.

Keywords Triglycerides $\cdot$ HDL-cholesterol $\cdot$ Sleep apnea syndrome $\cdot$ Non-overweight

\section{Introduction}

Recently, numerous studies have reported an independent association between insulin resistance and sleep apnea syndrome [1-5].

On the other hand, a high TG-HDL cholesterol ratio (TG-HDL) has been found to indicate insulin resistance in the general population [6].

However, no studies have reported on the association between TG-HDL and sleep apnea syndrome.

Furthermore, a previous Japanese study on sleep apnea syndrome reported a significant correlation between apnea index (AI) and body mass index (BMI), with the former being higher in participants with a BMI $\geq 25 \mathrm{~kg} / \mathrm{m}^{2}$ than in participants with a BMI $<25 \mathrm{~kg} / \mathrm{m}^{2}$ [7]. Another study reported a strong association between BMI and insulin resistance [8]. Therefore, the association between TG-HDL and sleep apnea syndrome should account for BMI status.

To investigate these associations, we conducted a crosssectional study of Japanese men undergoing screening for sleep apnea syndrome. 


\section{Materials and methods}

\section{Study populations}

The survey population comprised 1,558 men aged 30-69 referred for pulse oximetry at a sleep disorders clinic (Inoue Hospital, Nagasaki, Japan) from June 2012 to May 2013. Those from whom data (30) were not available were excluded, leaving 1,528 subjects participating in this study.

\section{Data collection and laboratory measurements}

Trained interviewers obtained information on medical history. Fasting blood samples were also obtained. Serum triglyceride (TG), serum HDL-cholesterol, serum aspartate aminotransferase (AST), serum alanine transaminase (ALT), serum $\gamma$-glutamyltranspeptidase $(\gamma$-GTP), serum creatinine, and fasting blood sugar were measured using standard laboratory procedures. Glomerular filtration rate (GFR) was estimated using an established method proposed by a working group of the Japanese Chronic Kidney Disease Initiative [9]. A pulse oximeter (PULSOX-Me300, KONICA MINOLTA, INC, Osaka, Japan) was used to measure $3 \%$ oxygen desaturation index (ODI), and sleep apnea syndrome was diagnosed as a $3 \%$ ODI of $\geq 15$ events $/ \mathrm{h}$, as described in a previous study [10], since a $3 \%$ ODI $\geq 15$ events/h was considered equivalent to an apneahypopnea index (AHI) $\geq 20$ events/h as determined by PSG, with a recommendation for treatment with CPAP for subjects showing AHI $\geq 20$ events/h [11-13].

\section{Statistical analyses}

Differences in age-adjusted mean values or prevalence of potential confounding factors by TG-HDL quartile were calculated using covariance or generalized linear models, and logistic regression models were used for calculating odds ratios (OR) and $95 \%$ confidence intervals (CI) to determine the association between sleep apnea syndrome and TG-HDL. In addition, subjects were stratified by BMI status. Because TG-HDL had a skewed distribution, logarithmic transformation was performed for calculating OR and $95 \% \mathrm{CI}$ to determine the association between sleep apnea syndrome and TG-HDL.

Three different approaches were used to make adjustments for confounding factors. First, data were adjusted only for age (Model 1). Next, further adjustment was made BMI (Model 2). Finally, other potential confounding factors were included, such as smoking status (never-smoker, former smoker, current smoker), alcohol consumption [non-drinker, current light drinker ( $<23 \mathrm{~g} /$ week), current moderate drinker (23-68 g/week), current heavy drinker
( $\geq 69 \mathrm{~g} /$ week)], systolic blood pressure, antihypertensive medication use, antidiabetic medication use, antihyperlipidemic medication use, serum aspartate aminotransferase (AST), serum $\gamma$-glutamyltranspeptidase $(\gamma$-GTP), fasting blood sugar, and glomerular filtration rate (GFR) (Model 3). Although diastolic blood pressure and ALT are known cardiovascular risk factors, they were not analyzed as confounding factors, since interaction between systolic blood pressure and diastolic blood pressure was $r=0.87$, $(p<0.001)$, and interaction between AST and ALT was $r=0.80(p<0.001)$.

All statistical analyses were performed with the SAS system for Windows (version 9.3; SAS Inc., Cary, NC). $p$ values $<0.05$ were regarded as being statistically significant.

\section{Ethical considerations}

This study was approved by the Ethics Committee for Human Use of Nagasaki University (project registration number 15033077). Written consent forms were available in Japanese to ensure comprehensive understanding of the study objectives, and informed consent was provided by the participants.

\section{Results}

\section{Characteristics of the study populations}

Among the survey population comprised of 1,528 men, 971 had a BMI $<25 \mathrm{k} / \mathrm{m}^{2}$ and 557 had a BMI $\geq 25 \mathrm{~kg} / \mathrm{m}^{2}$.

Table 1 shows the age-adjusted characteristics of the study populations for total subjects stratified by BMI status.

We also found that participants with a BMI $\geq 25 \mathrm{~kg} / \mathrm{m}^{2}$ had significantly higher values for fasting blood sugar and TG-HDL than participants with a BMI $<25 \mathrm{~kg} / \mathrm{m}^{2}$. Ageadjusted values for fasting blood sugar were 100 and 107 in patients with a BMI $<25 \mathrm{~kg} / \mathrm{m}^{2}$ and a BMI $\geq 25 \mathrm{~kg} / \mathrm{m}^{2}$, respectively $(p<0.001)$; and for TG-HDL were 2.06 and 3.26 in patients with a BMI $<25 \mathrm{~kg} / \mathrm{m}^{2}$ and a BMI $\geq 25 \mathrm{~kg} / \mathrm{m}^{2}$, respectively $(p<0.001)$.

\section{Associations between TG-HDL and sleep apnea syndrome}

Table 2 shows the associations between TG-HDL and sleep apnea syndrome in total subjects stratified by BMI status. For total subjects, with the lowest quartile of TGHDL as reference group, the age-adjusted OR of sleep apnea syndrome for the highest quartile of TG-HDL was 5.63 (95\% CI: 3.48-9.10). After further adjustment for BMI, this association slightly weakened but remained 


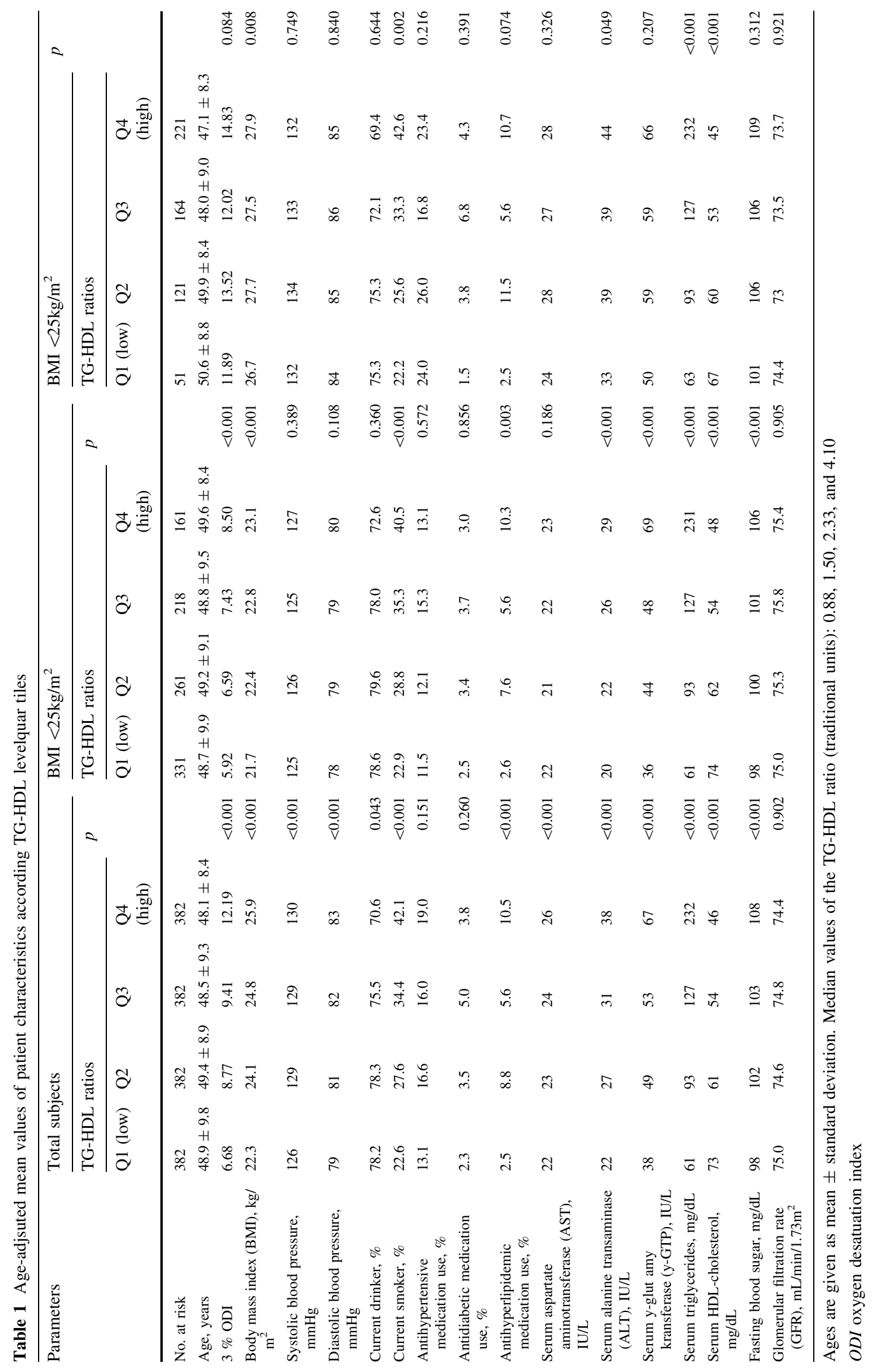


Table 2 Odds ratios (OR) and $95 \%$ confidence intervals (CI) for sleep apnea syndrome

\begin{tabular}{|c|c|c|c|c|c|c|}
\hline & \multicolumn{4}{|c|}{ TG-HDL ratios quartiles } & \multirow[t]{2}{*}{$p$ for trend } & \multirow[t]{2}{*}{ Log TG-HDL ratic } \\
\hline & Q1 (Low) & Q2 & Q3 & Q4 (High) & & \\
\hline \multicolumn{7}{|l|}{ Total subjects } \\
\hline No. at risk & 382 & 382 & 382 & 382 & & \\
\hline No. of cases $(\%)$ & $23(6.0)$ & $51(13.4)$ & $67(17.5)$ & $100(26.2)$ & & \\
\hline Model 1 & 1.00 & $2.40(1.43-4.01)$ & $3.35(2.04-5.51)$ & $5.63(3.48-9.10)$ & $<0.001$ & $2.24(1.83-2.75)$ \\
\hline Model 2 & 1.00 & $1.39(0.81-2.39)$ & $1.70(1.00-2.88)$ & $2.19(1.30-3.69)$ & 0.001 & $1.46(1.15-1.85)$ \\
\hline Model 3 & 1.00 & $1.38(0.80-2.39)$ & $1.73(1.01-2.96)$ & $2.24(1.31-3.88)$ & 0.001 & $1.49(1.17-1.92)$ \\
\hline \multicolumn{7}{|c|}{ Subjects with a BMI $<25 \mathrm{~kg} / \mathrm{m}^{2}$} \\
\hline No. at risk & 331 & 261 & 218 & 161 & & \\
\hline No. of cases (percentage) & $11(3.3)$ & $17(6.5)$ & $22(10.1)$ & $23(14.3)$ & & \\
\hline Model 1 & 1.00 & $2.02(0.92-4.38)$ & $3.28(1.55-6.93)$ & $4.82(2.28-10.18)$ & $<0.001$ & $2.11(1.49-2.99)$ \\
\hline Model 2 & 1.00 & $1.65(0.75-3.64)$ & $2.51(1.17-5.38)$ & $3.41(1.57-7.39)$ & $<0.001$ & $1.79(1.24-2.59)$ \\
\hline Model 3 & 1.00 & $1.77(0.79-3.94)$ & $2.65(1.22-5.77)$ & $4.20(1.85-9.50)$ & $<0.001$ & $2.03(1.36-3.03)$ \\
\hline \multicolumn{7}{|c|}{ Subjects with a $\mathrm{BMI} \geq 25 \mathrm{~kg} / \mathrm{m}^{2}$} \\
\hline No. at risk & 51 & 121 & 164 & 221 & & \\
\hline No. of cases $(\%)$ & $12(23.5)$ & $34(28.1)$ & $45(27.4)$ & $77(34.8)$ & & \\
\hline Model 1 & 1.00 & $1.28(0.60-2.74)$ & $1.26(0.60-2.63)$ & $1.80(0.89-3.65)$ & 0.054 & $1.41(1.05-1.88)$ \\
\hline Model 2 & 1.00 & $0.97(0.44-2.13)$ & $1.01(0.47-2.16)$ & $1.30(0.62-2.71)$ & 0.250 & $1.25(0.92-1.69)$ \\
\hline Model 3 & 1.00 & $0.95(0.42-2.12)$ & $1.04(0.48-2.27)$ & $1.25(0.58-2.68)$ & 0.313 & $1.23(0.89-1.70)$ \\
\hline
\end{tabular}

Model 1 adjusted for age only, Model 2 adjusted further for body mass index (BMI), Model 3 adjusted further for systolic blood pressure, alcohol intake, smoking, antihypertensive medication use, antidiabetic medication use, antihyperlipidemic medication use, serum aspartate aminotransferase (AST), serum $\gamma$-glutamyltranspeptidase ( $\gamma$-GTP), fasting blood sugar, and glomerular filtration rate (GFR). Median values of the TGHDL ratio (traditional units) were $0.88,1.50,2.33$, and 4.10

significant; the adjusted-OR for the corresponding value was 2.19 (95\% CI: 1.30-3.69). However, after further adjustment for other classical cardiovascular risk factors, the magnitude remained the same at 2.24 (95\% CI: 1.31-3.88). We also found that these significant associations were limited to participants with a lower BMI $\left(<25 \mathrm{~kg} / \mathrm{m}^{2}\right)$. Further analysis showed a risk of sleep apnea syndrome in participants with a higher BMI status. Compared to participants with a BMI $<25 \mathrm{~kg} / \mathrm{m}^{2}$, the age-adjusted OR of sleep apnea syndrome in participants with a $\mathrm{BMI} \geq 25 \mathrm{~kg} / \mathrm{m}^{2}$ was $5.41(95 \% \quad \mathrm{CI}$ : 4.01-7.31; $p<0.001)$.

\section{Interaction between TG-HDL level and two BMI categories on sleep apnea syndrome}

Since our study population was compromised of nonoverweight ( $n=971)$ and overweight $(n=557)$ subjects, to avoid the influence of sample size bias on the correlation between TG-HDL and sleep apnea syndrome, we evaluated the interaction between TG-HDL levels and two BMI categories on sleep apnea syndrome. An investigation of the effects of interaction between TG-HDL level and two BMI categories $\left(\mathrm{BMI} \geq 25 \mathrm{~kg} / \mathrm{m}^{2}\right.$ and $\mathrm{BMI}<25 \mathrm{~kg} / \mathrm{m}^{2}$ ) on sleep apnea syndrome revealed significant interaction between TG-HDL level and BMI category, with an ageadjusted $\mathrm{p}$ value for the effect of this interaction on sleep apnea syndrome of $p=0.040$.

\section{Correlation between fasting blood sugar and $3 \%$ ODI by BMI status}

We also evaluated the correlation between blood sugar and $3 \%$ ODI by BMI status, and found that the fasting blood sugar correlated with $3 \%$ ODI in participants with a BMI $<25 \mathrm{~kg} / \mathrm{m}^{2}$, but not a BMI $\geq 25 \mathrm{~kg} / \mathrm{m}^{2}$ with a simple correlation coefficient (Pearson) and $p$ value of $r=0.15$ $(p<0.001)$ in participants with a BMI $<25 \mathrm{~kg} / \mathrm{m}^{2}$, and $r=0.03(p=0.533)$ in participants with a BMI $\geq 25 \mathrm{~kg} /$ $\mathrm{m}^{2}$ (Table 3).

\section{Discussion}

A major finding of the present study was a positive association between TG-HDL level and sleep apnea syndrome in Japanese men with a lower BMI $\left(<25 \mathrm{~kg} / \mathrm{m}^{2}\right)$ but not with a higher BMI $\left(\geq 25 \mathrm{~kg} / \mathrm{m}^{2}\right)$. 
Table 3 Correlation between fasting blood sugar and $3 \%$ ODI

Simple correlation coefficient

Subjects with a BMI $<25 \mathrm{~kg} / \mathrm{m}^{2}$

$r=0.15(p<0.001)$

Subjects with a BMI $\geq 25 \mathrm{~kg} / \mathrm{m}^{2}$

$r=0.03(p=0.533)$

A previous case-control cross-sectional study with nonobese subjects reported that obstructive sleep apnea syndrome is independently associated with dyslipidemia [14]. Additionally, another study reported that respiratory disturbance index is positively correlated with triglycerides and inversely correlated with HDL-cholesterol among participants aged $<65$ years, but not participants aged $\geq 65$ years [15]. These studies are compatible with our present study showing that TG-HDL is significantly positively associated with sleep apnea syndrome. Further, we found additional evidence that this positive association was limited to non-obese subjects.

Since our present study investigated the association between TG-HDL and sleep apnea syndrome, the impact of triglycerides, HDL-cholesterol, and TG-HDL on sleep apnea syndrome is important. Conduction of additional analyses after dividing the values for triglycerides, HDLcholesterol, and TG-HDL into quartiles separately showed that sleep apnea syndrome had no linear association with triglycerides and HDL, while a linear association was observed for TG-HDL. Among participants with BMI $<25 \mathrm{~kg} / \mathrm{m}^{2}$, in the reference group of the lowest quartile (Q1) of triglycerides, the multivariable ORs of sleep apnea syndrome were $1.29(0.58-2.86)$ for Q2, 2.84 (1.37-5.90) for Q3, and 2.59 (1.16-5.77) for Q4. Additionally, in the reference group of the lowest quartile (Q1) of HDL-cholesterol, the multivariable ORs of sleep apnea syndrome were $0.63(0.32-1.26)$ for $\mathrm{Q} 2,0.71(0.35-1.44)$ for Q3, and $0.41(0.19-0.89)$ for $\mathrm{Q} 4$, while in the reference group of the lowest quartile (Q1) of TG-HDL, the multivariable ORs of sleep apnea syndrome were 1.77 (0.79-3.94) for $\mathrm{Q} 2,2.65$ (1.22-5.77) for $\mathrm{Q} 3$, and 4.20 (1.85-9.50) for Q4. Accordingly, instead of triglycerides and HDL, the ratio of TG-HDL should be used to evaluate the risk of sleep apnea syndrome.

TG-HDL has been found to be correlated with insulin resistance [16-18], as has sleep apnea syndrome [1-5]. Furthermore, the Sleep Heart Health Study of community dwelling subjects reported that those with sleep-disordered breathing (respiratory disturbance index (RDI) of $\geq 15$ events/h) have higher values for the homeostasis model assessment (HOMA) index than participants with an RDI $<5.0$ and an RDI 5.0-14.9 [5]. This study is compatible with our results showing a significant positive association between TG-HDL and sleep apnea syndrome. Although insulin resistance may play an important role in the correlation between TG-HDL and sleep apnea syndrome, the underlying mechanism of this correlation is not yet clarified. Sleep apnea syndrome leads to several physiological disturbances such as intermitted hypoxia, sleep fragmentation, and an increase in autonomic tone associated with insulin resistance [19]. Insulin resistance is correlated with reduced lipoprotein lipase (LPL) activity which results in hypertriglyceridemia and decreased synthesis of HDL-cholesterol [20].

We also found that the positive association between TGHDL and sleep apnea syndrome is limited to participants with a BMI $<25 \mathrm{~kg} / \mathrm{m}^{2}$. Although we did not record insulin data, fasting blood sugar was available. Since insulin resistance should positively correlate with fasting blood sugar, the correlation between fasting blood sugar and $3 \%$ ODI may partly indicate the correlation between insulin resistance and $3 \%$ ODI. Additional analyses showed that fasting blood sugar correlates with $3 \%$ ODI in participants with a BMI $<25 \mathrm{~kg} / \mathrm{m}^{2}$ but not a BMI $\geq 25 \mathrm{~kg} / \mathrm{m}^{2}$. This partially supports a mechanism in which insulin resistance is significantly associated with $3 \%$ ODI only among participants with a BMI $<25 \mathrm{~kg} / \mathrm{m}^{2}$. Therefore, compared to participants with a $\mathrm{BMI}<25 \mathrm{~kg} / \mathrm{m}^{2}$, those with a BMI $\geq 25 \mathrm{~kg} / \mathrm{m}^{2}$ might have a higher risk of sleep apnea syndrome independent of insulin resistance-even stronger insulin resistance is observed in those with a BMI $\geq 25 \mathrm{~kg}$ / $\mathrm{m}^{2}$ than those with a BMI $<25 \mathrm{~kg} / \mathrm{m}^{2}$. In fact, participants in this study with a BMI $\geq 25 \mathrm{~kg} / \mathrm{m}^{2}$ showed significantly higher values for fasting blood sugar and TG-HDL than those with a BMI $<25 \mathrm{~kg} / \mathrm{m}^{2}$.

Thus, the former group may be masked by these risk factors that are independent of TG-HDL.

Potential limitations in this study warrant consideration. Although $3 \%$ ODI is considered to be an initial diagnostic test for sleep apnea syndrome, it should not on its own be considered as conclusive evidence of sleep apnea syndrome in subjects with a BMI below $25 \mathrm{~kg} / \mathrm{m}^{2}$ [21]. However, the diagnostic sensitivity of each of the $3 \%$ ODI thresholds for sleep apnea syndrome increased with BMI, while at the same time, the specificity of ODI for sleep apnea syndrome fell [21]. This study is compatible with our additional analysis with our study population of 219 subjects $\left(82\right.$ for BMI $<25 \mathrm{kgm}^{2}$ and 137 for $\mathrm{BMI} \geq 25 \mathrm{~kg} / \mathrm{m}^{2}$ ) who had received overnight recordings of both PULSOXMe300 and standard polysomnography (PSG). The sensitivity and specificity were 62 and $63 \%$ for subjects with BMI $<25 \mathrm{~kg} / \mathrm{m}^{2}$ and 77 and $50 \%$ for subjects with BMI $\geq 25 \mathrm{~kg} / \mathrm{m}^{2}$ ) for detecting an AHI of $20 \geq$ events $/ \mathrm{h}$ by PSG using a cut-off threshold of $3 \%$ ODI $=15$. Although the correlation between TG-HDL and sleep apnea syndrome was shown to be independent of the traditional risk 
factors, we did not adjust for other potential confounding factors whose values were associated with sleep apnea syndrome [22] and dyslipidemia [23] such as advanced glycation end-products. Further investigation using data from advanced glycation end-products is necessary.

Because this study was a cross-sectional, no causal relationships were able to be established.

In conclusion, an independent positive association between the TG-HDL levels and the risk of sleep apnea syndrome was observed in participants with a BMI of $<25 \mathrm{~kg} / \mathrm{m}^{2}$ but not for participants with a BMI $\geq 25 \mathrm{~kg}$ / $\mathrm{m}^{2}$. TG-HDL levels could be an efficient tool to estimate the risk of sleep apnea syndrome in non-overweight, but not in overweight, Japanese men. Since Japanese subjects with sleep apnea syndrome are reported to have a lower BMI than Caucasian subjects, our findings might be particularly applicable to the former group [24].

Acknowledgments The research did not receive financial support.

\section{Compliance with ethical standards}

Conflicts of interest The authors declare that they have no conflicts of interest.

Human and animal rights and informed consent All procedures performed in studies involving human participants were in accordance with the ethical standards of the institution research committee and with the 1964 Helsinki declaration and its later amendments or comparable ethical standards. The Ethics Committee for Human Use of Nagasaki University obtained ethical approval.

\section{References}

1. West SD, Nicoll DJ, Stradling JR. Prevalence of obstructive sleep apnoea in men with type 2 diabetes. Thorax. 2006;61:945-50.

2. Sulit L, Storfer-Isser A, Kirchner HL, Redline S. Differences in polysomnography predictors for hypertension and impaired glucose tolerance. Sleep. 2006;29:777-83.

3. Makino S, Handa H, Suzukawa K, Fujiwara M, Nakamura M, Muraoka S, Takasago I, Tanaka Y, Hashimoto K, Sugimoto T. Obstructive sleep apnoea syndrome, plasma adiponectin levels, and insulin resistance. Clin Endocrinol (Oxf). 2006;64:12-9.

4. Hassaballa HA, Tulaimat A, Herdegen JJ, Mokhlesi B. The effect of continuous positive airway pressure on glucose control in diabetic patients with severe obstructive sleep apnea. Sleep Breath. 2005;9:176-80.

5. Punjabi NM, Shahar E, Redline S, Gottlieb DJ, Givelber R, Resnick HE, Sleep Heart Health Study Investigators. Sleep-disordered breathing, glucose intolerance, and insulin resistance: the Sleep Heart Health Study. Am J Epidemiol. 2004;160:521-30.

6. González-Chávez A, Simental-Mendía LE, Elizondo-Argueta S. Elevated triglycerides/HDL-cholesterol ratio associated with insulin resistance. Cir Cir. 2011;79:126-31.

7. Suzuki R, Akashiba T, Saito O, Horie T. Risk factors in development of sleep apnea syndrome-a large population study using a simple monitoring system. Nihon Kokyuki Gakkai Zasshi. 2002;40:653-9 (Article in Japanese).
8. Colhoun HM. The big picture on obesity and insulin resistance. J Am Coll Cardiol. 2002;40:944-5.

9. Imai E. Equation for estimating GFR from creatinine in Japan. Nihon Rinsho. 2008;66:1725-9 (Article in Japanese).

10. Ishimaru N, Maeno T, Suzuki M, Maeno T. Prevalence of sleep apnea syndrome in Japanese patients with persistent fatigue. Gen Med. 2012;13:103-9.

11. Tanaka S, Shima M. Assessment of screening tests for sleep apnea syndrome in the workplace. J Occup Health. 2010;52:99-105.

12. Meoli AL, Casey KR, Clark RW, Coleman JA Jr, Fayle RW, Troell RJ, Iber C, Clinical Practice Review Committee. Hypopnea in sleep-disordered breathing in adults. Sleep. 2001;24:469-70.

13. Fietze I, Dingli K, Diefenbach K, Douglas NJ, Glos M, Tallafuss M, Terhalle W, Witt C. Night-to-night variation of the oxygen desaturation index in sleep apnoea syndrome. Eur Respir J. 2004;24:987-93.

14. Lin QC, Zhang XB, Chen GP, Huang DY, Din HB, Tang AZ. Obstructive sleep apnea syndrome is associated with some components of metabolic syndrome in nonobese adults. Sleep Breath. 2012;16:571-8.

15. Newman AB, Nieto FJ, Guidry U, Lind BK, Redline S, Pickering TG, Quan SF, Sleep Heart Health Study Research Group. Relation of sleep-disordered breathing to cardiovascular disease risk factors: the Sleep Heart Health Study. Am J Epidemiol. 2001;154:50-9.

16. McLaughlin T, Abbasi F, Cheal K, Chu J, Lamendola C, Reaven G. Use of metabolic markers to identify overweight individuals who are insulin resistant. Ann Intern Med. 2003;139:802-9.

17. Karelis AD, Pasternyk SM, Messier L, St-Pierre DH, Lavoie JM, Garrel D, Rabasa-Lhoret R. Relationship between insulin sensitivity and the triglyceride-HDL-C ratio in overweight and obese postmenopausal women: a MONET study. Appl Physiol Nutr Metab. 2007;32:1089-96.

18. Tangvarasittichai S, Poonsub P, Tangvarasittichai O. Association of serum lipoprotein ratios with insulin resistance in type 2 diabetes mellitus. Indian J Med Res. 2010;131:641-8.

19. Rajan P, Greenberg H. Obstructive sleep apnea as a risk factor for type 2 diabetes mellitus. Nat Sci Sleep. 2015;7:113-25.

20. Shimada M, Ishibashi S, Gotoda T, Kawamura M, Yamamoto K, Inaba T, Harada K, Ohsuga J, Perrey S, Yazaki Y, Yamada N. Overexpression of human lipoprotein lipase protects diabetic transgenic mice from diabetic hypertriglyceridemia and hypercholesterolemia. Arterioscler Thromb Vasc Biol. 1995;15:1688-94.

21. Ling IT, James AL, Hillman DR. Interrelationships between body mass, oxygen desaturation, and apnea-hypopnea indices in a sleep clinic population. Sleep. 2012;35:89-96.

22. Lam JC, Tan KC, Lai AY, Lam DC, Ip MS. Increased serum levels of advanced glycation end-products is associated with severity of sleep disordered breathing but not insulin sensitivity in non-diabetic men with obstructive sleep apnoea. Sleep Med. 2012;13:15-20.

23. Bucala R, Makita Z, Vega G, Grundy S, Koschinsky T, Cerami A, Vlassara H. Modification of low density lipoprotein by advanced glycation end products contributes to the dyslipidemia of diabetes and renal insufficiency. Proc Natl Acad Sci USA. 1994;91:9441-5.

24. Genta PR, Marcondes BF, Danzi NJ, Lorenzi-Filho G. Ethnicity as a risk for obstructive sleep apnea: comparison of Japanese descendants and white males in São Paulo, Brazil. Braz J Med Biol Res. 2008;41:728-33. 